\section{Chemotherapy and breast conservative surgery as a safe alternative to mastectomy}

Preoperative treatment of breast tumors with primary chemotherapy (PC) could reduce tumor size and allow breast conservative surgery (BCS) to be applied to patients who would otherwise be candidates for mastectomy. Gentilini et al. have assessed the ipsilateral breast tumor reappearance (IBTR) rate in 309 women who presented with either locally advanced or large monofocal operable breast cancer. After PC, 195 (63.1\%) of these patients were identified as suitable for BCS and were operated on.

Thirteen $(6.7 \%)$ of the women who received BCS had an IBTR during a follow-up ranging from 7 to 90 months. The authors consider this to be an acceptably low rate of IBTR. They do, however, note that their sample of BCS recipients exhibited a high rate of positive margins at final pathologic report $(24 / 195 ; 12.3 \%)$ and that this is an expected risk of conservative surgery following PC. Presence of positive margins was associated with a higher risk of IBTR after 3 years of follow-up than absence of these margins $(13.3 \%$ vs $4.7 \% ; P=0.05)$.

Despite their association with IBTR, positive margins did not significantly affect overall survival during a short-term follow-up. The authors suggest that PC followed by BCS should be discussed with patients as a potential treatment option. If successful, the procedure could allow breast conservation without worsening overall survival, and without undue risk of IBTR.

Original article Gentilini $\mathrm{O}$ et al. (2006) Ipsilateral breast tumor reappearance in patients treated with conservative surgery after primary chemotherapy: the role of surgical margins on outcome. J Surg Oncol 94: 375-379

\section{Disease relapse is unlikely in patients with slowly rising PSA levels following treatment}

The clinical significance of fluctuating serum prostate-specific antigen (PSA) levels following definitive external beam radiotherapy (EBRT) for prostate cancer is unclear. In addition, PSA oscillations in patients treated with neoadjuvant androgen deprivation therapy (ADT) before EBRT are not well characterized. To address these issues, Zelefsky et al. undertook a retrospective study of patients treated with EBRT alone or in combination with short-term ADT.

The investigators identified 570 patients who met the inclusion criteria of $>10$ consecutive PSA measurements taken during follow-up (median 103 months). In patients receiving ADT (median 3 months) before radiotherapy $(n=194)$, three distinct PSA patterns were documented: a stable trend was seen in $44 \%$, a rising trend followed by stabilization in $25 \%$, and a rising trend in $31 \%$. In the second of these groups, stabilization was reached at a median PSA level of $0.9 \mathrm{ng} / \mathrm{ml}$ and PSA remained durably repressed. The incidence of distant metastasis was higher in patients whose PSA levels did not stabilize, but distant-metastasis-free survival was similar in individuals exhibiting the other two trends. In patients receiving EBRT alone $(n=376)$, PSA levels either declined and then stabilized, or declined and then slowly or rapidly increased. In this cohort, patients exhibiting rapidly rising PSA levels had a higher risk of developing distant metastases. The authors conclude that slowly rising PSA levels following treatment do not indicate clinically significant disease. Elevations of PSA are common after neoadjuvant $A D T$ plus radiotherapy - consistent with testosterone renormalizing - and often the levels then stabilize.

Original article Zelefsky MJ et al. (2006) Evaluation of postradiotherapy PSA patterns and correlation with 10 -year disease free survival outcomes for prostate cancer. Int $J$ Radiat Oncol Biol Phys 66: 382-388

\section{Gene expression profiling can predict the outcome of stage II colon cancer patients}

Gene expression profiling is a powerful method used widely in the prognosis prediction (PP) of various types of cancer, including stage II colon cancer. A previous study identified a 23-gene prognosis signature (PS) with a $78 \%$ PP accuracy, as validated by analysis of an independent set of patients.

In order to identify and validate the performance of a microarray-based PP as well as the performance of the previously identified 23-gene PS, Barrier and co-authors used oligonucleotide microarrays to profile mRNA samples extracted from tumors removed from 50 patients with stage II colon adenocarcinoma. Of these patients, who had not received any 